\title{
Metabolic Equivalents of Selected Sedentary and Physical Activities in Chinese Youth
}

\author{
Yong Gao, Haichun Sun, Jie Zhuang, Jian Zhang, \\ Lynda Ransdell, Zheng Zhu, and Siya Wang
}

\begin{abstract}
Background: This study determined the metabolic equivalents (METs) of several activities typically performed by Chinese youth. Methods: Thirty youth (12 years) performed 7 activities that reflected their daily activities while Energy Expenditure (EE) was measured in a metabolic chamber. Results: METs were calculated as activity EE divided by participant's measured resting metabolic rate. A MET value ranging from 0.8 to 1.2 was obtained for sleeping, watching TV, playing computer games, reading and doing homework. Performing radio gymnastics had a MET value of 2.9. Jumping rope at low effort required 3.1 METs. Except for watching TV, METs for other activities in this study were lower than Youth Compendium values. Conclusions: The results provide empirical evidence for more accurately assessing EE of activities commonly performed by Chinese youth. This is the first study to determine METs for radio gymnastics and jump rope in Chinese youth.
\end{abstract}

Keywords: energy expenditure, obesity, metabolic chamber, radio gymnastics, jump rope

During the past 3 decades, Chinese youth have experienced a dramatic rise in obesity, increasing from $0.1 \%$ in 1985 to $5.0 \%$ in $2010 .{ }^{1}$ Although obesity is caused by many factors, physical inactivity has been identified as one of the major contributors. To promote physical activity (PA) participation, the World Health Organization recommended that children and adolescents aged 5 to 17 years should accumulate at least 60 minutes of moderate-to-vigorous physical activity (MVPA) daily. ${ }^{2}$ However, most Chinese youth do not meet these PA recommendations, and obesity rates in China have continued to increase. ${ }^{3}$ Therefore, it is of vital importance to promote MVPA participation among Chinese youth for optimal health benefits.

Youth in China are under great pressure to achieve scholastically and often have to spend much of their time doing homework. In addition, due to many factors (eg, lack of time, space, resources, poor air quality, etc.), common leisure activities for Chinese youth are sedentary (eg, reading, watching TV, and playing computer games). The primary source of PA for Chinese youth is schoolrelated activities (ie, before/during/after school activities provided by a school using school facilities); participation in MVPA outside of school is almost nonexistent. ${ }^{4}$

In China, every school day, students are required to participate in a 5-minute school-wide radio gymnastics program during morning recess time. Radio gymnastics, first launched nationally in 1951, are considered mandatory PA in schools. Radio gymnastics are a predetermined routine of calisthenics that are done to music, with the idea that students will participate in physical activity using a

Gao is with the Dept of Kinesiology, Boise State University. Sun is with the School of Physical Education and Exercise Science, University of South Florida. Zhuang (correspondence: zhuangjiesh@163.com) is with the Dept of Kinesiology; Zhang is with the Dept of Applied Exercise Psychology; Zhu and Wang are with the Dept of Exercise Sciences; Shanghai University of Sport, China. Ransdell is with the College of Health Solutions, Arizona State University. systematic and standardized routine, in an effort to build group cohesion, and enhance energy levels and fitness. ${ }^{5}$ This preprogrammed style makes it easy for classroom teachers to deliver the activity, and it provides a mandatory activity break for students and teachers. Research on school-based radio gymnastics has mainly focused on historical development, policy-related implementation strategies, and program design. ${ }^{6}$ Little is known about its energy cost, which makes it difficult to devise evidence-based strategies for implementing these exercise programs.

Another school-based activity, in which many Chinese students participate, is an afterschool program that combines free play and exercises. The majority of Chinese youth $(87.4 \%)$ participate in jump rope as part of an afterschool program at least once a week. ${ }^{7}$ To ensure that Chinese students achieve satisfactory fitness levels, the Chinese Department of Education ${ }^{8}$ has established national standards for many exercises, including standards for jump rope for 6th graders/12 years of age. Unfortunately, the energy expenditure (EE) of jump roping in Chinese youth is unknown. Although metabolic equivalents (METs) of jump rope are available from the Compendium of Energy Expenditures for Youth, ${ }^{9}$ the Youth Compendium uses data collected from individuals in western countries, and there are demonstrated differences in metabolic rates based on racial/ethnic background. ${ }^{10}$ Specifically, when young white men and women were compared with their Asian counterparts, absolute resting energy expenditure (REE) was lower in Asians than in whites, an effect that was mitigated when REE was corrected for fat-free mass. ${ }^{10}$ Therefore, using METs from the Youth Compendium to assess EE for Chinese youth may result in substantial errors.

Given the need to establish appropriate MET values for a variety of activities in which Chinese youth participate, an effort has been recently started by a research team in a top sport university in China to develop a Chinese-specific Youth Compendium. This study was one of the first to determine EE and METs of several sedentary and physical activities typically performed by 12 -yearold Chinese youth. 


\section{Methods}

\section{Participants}

Thirty Chinese youth (18 boys and 12 girls; 12 years of age) were recruited. This age group was selected because it is representative of the elementary to middle school transitional age group, for which there is a dearth of research in PA energy expenditure in Chinese populations. In addition, PA participation declines dramatically at this age, so it is important to accurately quantify PA that does exist because such information can be valuable for intervention programs intended to improve PA participation and health outcomes among this age group of students in China. To be included in this study, participants had to be healthy, willing to stay in a metabolic chamber for 16 hours, and able to perform the examined activities with no limitations. The study was approved by the Ethics Advisory Committee of the Shanghai University of Sport, China. The participant assented to participate and his/her parents/legal guardians signed an informed consent before data collection.

\section{Measurements}

The participants' height (in $\mathrm{cm}$ ) and weight (in $\mathrm{kg}$ ) were measured twice with clothes/without shoes using an automatic height and body composition Analyzer (Tanita, DC-250). When 2 measurements did not agree, then 2 additional measures were taken until there was an agreement. EE was measured using open-circuit indirect room calorimetry. The metabolic chamber (Fuji Human Calorimeter model: FHC-20S) was an airtight room (24000 L) equipped with a bed, desk, chair, telephone, toilet, and sink. The temperature and relative humidity in the room were controlled at $25^{\circ} \mathrm{C}$ and $55 \%$, respectively. The oxygen and carbon dioxide concentrations of the air supply and exhaust were measured using mass spectrometry. The gas analyzer was calibrated using 4 certified gas mixtures. The flow rate exhausted from the chamber was measured using a Yamatake-CMS0200 (Phoenix, AZ). Minute-by-minute oxygen consumption and carbon dioxide production were collected and converted to energy equivalents using Weir's equation. ${ }^{11}$

\section{Procedures}

Table 1 contains a summary of the 18 hours of activities. Participants had several options for diet for breakfast and for dinner, respectively, with the nutritional composition being similar (ie, approximately $18 \%$ fat, $15 \%$ protein, and $67 \%$ carbohydrates) in each meal.

To measure resting metabolic rate (RMR; 4 hours after dinner) and basal metabolic rate (BMR; after 8 hours sleep), participants were instructed to lie in bed in the supine position. EE was then measured for 40 minutes with the subject quiet, but awake. The first 10 and last 5 minutes of measurements were eliminated. RMR and BMR were obtained for 25 minutes, respectively.

Several activities, similar to typical daily activities performed by Chinese youth (ie, doing homework, reading, watching TV, playing computer games, performing radio gymnastics, and jump roping) were selected for this study. EE during homework, reading, playing computer games, and watching TV was measured twice, once in the morning and once in the evening, for 30 minutes per session. TV programs and computer games were self-selected by the participant. Data from the first 9 minutes and last minute of each sedentary activity were eliminated.

EE during radio gymnastics was measured for 9 minutes. Data from the first 2 minutes and the last minute were eliminated. EE during jumping rope was measured continuously for 8 minutes with 45 to 50 turns per minute (Note: 45 to 50 turns per minute was the national standard established for 12 year old Chinese students jumping rope) ${ }^{8}$ Only data that accumulated 45 to 50 turns per minute were included for data analysis.

\section{Data Analysis}

MET values were calculated as activity EE divided by each participant's measured RMR. Descriptive statistics were calculated for all variables. One sample $t$-tests were conducted using SPSS 22 to determine whether significant differences existed between the METs obtained in this study and those listed on the Youth Compendium.

\section{Results}

Table 2 presents sample characteristics. Table 3 presents EE and METs by gender for this study and from the Youth Compendium. METs obtained from the current study were slightly lower than the Youth Compendium METs for sleeping $(P<.005)$, doing homework $(P<.005)$, and reading $(P<.01)$; but large MET differences were observed for playing computer games $(P<.005)$ and jumping rope $(P<.005)$. Performing radio gymnastics had a MET value classified as light intensity.

\section{Discussion}

In this study, METs for sleeping, homework, reading, TV, computer games in Chinese youth were around 1. Performing radio gymnastics was considered a light intensity PA, and jumping rope at low effort required 3 METs. Although there was no difference in METs for watching TV, METs for other activities were lower than the METs from the Youth Compendium.

METs from the Youth Compendium may slightly overestimate EE for several sedentary activities, but could considerably overestimate EE for jumping rope in Chinese youth. The MET value of playing computer games in this study was much lower than the Compendium value. This study allowed participants to play computer games of their choice, so it is possible that participants chose a game with which they were familiar-which meant they could play it more efficiently, expending less energy.

Radio gymnastics is a unique, mandated morning exercise routine in almost every elementary/secondary school across China, and its EE/METs have never been reported. In this study, the MET value of performing radio gymnastics falls into the light intensity PA category. Given that millions of Chinese youth perform radio gymnastics every school day for at least 5 minutes, MET information obtained in this study may have an impact on predicting PA levels in Chinese children. One of the major intents of mandating radio gymnastics in school in China is to promote PA and fitness, ${ }^{5}$ however, the effects of radio gymnastics have not yet been fully evaluated. For optimal health outcomes, performing this light intensity exercise 5 minutes per school day is likely not enough. Therefore, conducting preliminary examinations of the impact of these 5-minute segments is important to determine whether longer, higher intensity, or more frequent radio gymnastics segments are desired to enhance health or fitness benefits.

Jump rope is popular and China has national standards to assess student jump rope performance (eg, 6th graders, 12 years old, need to perform 45 to 50 turns per minute to receive a passing score) ${ }^{8}$ 
Table 1 Schedule of Activities During 18-Hour Stay in the Metabolic Chamber

\begin{tabular}{|c|c|c|}
\hline Times & Activity & Contents \\
\hline $17: 00-17: 30$ & Anthropometric Examination & Informed consent, measuring height, weight, heart rate, etc. \\
\hline $17: 30-18: 00$ & Shower and change clothes & \\
\hline $18: 00-18: 30$ & Dinner & \\
\hline $18: 40$ & $\begin{array}{l}\text { Enter the room and start } \\
\text { protocol }\end{array}$ & $\begin{array}{l}\text { Familiarize with the room environment, prohibited from playing computer games and } \\
\text { strenuous PA }\end{array}$ \\
\hline 19:00 & Start recording the data & \\
\hline 19:00-19:20 & Preparation & \\
\hline $19: 20-19: 50$ & Sedentary Behavior & Homework/Writing (sitting) \\
\hline 19:50-20:20 & Sedentary Behavior & Watching TV (sitting) \\
\hline 20:20-21:00 & Lying on bed & Stay awake, quiet, no PA \\
\hline $21: 00-21: 30$ & Sedentary Behavior & Playing computer games (sitting) \\
\hline 21:30-22:00 & Sedentary Behavior & Reading (sitting) \\
\hline 22:00-22:10 & Wash & \\
\hline $22: 10-22: 50$ & Lying on bed & Stay awake, quiet, in supine position (measurement of resting metabolic rate \\
\hline 22:50-23:00 & Presleep & Prepare for sleep \\
\hline 23:00-07:00 & Sleep & Sleeping ( 8 hours) \\
\hline 07:00-07:40 & Lying on bed & Stay awake, quiet, in supine position (measurement of basal metabolic rate) \\
\hline 07:40-08:00 & Breakfast & \\
\hline 08:00-08:40 & Random activity & Writing, watching TV, or reading, prohibited from playing computer games and strenuous PA \\
\hline 08:40-09:10 & Sedentary Behavior & Homework/Writing (sitting) \\
\hline 09:10-09:40 & Sedentary Behavior & Watching TV (sitting) \\
\hline 09:40-10:10 & Sedentary Behavior & Playing computer games (sitting) \\
\hline 10:10-10:40 & Sedentary Behavior & Reading (sitting) \\
\hline 10:40-10:50 & Radio gymnastics & Performing radio gymnastics twice for 9 min \\
\hline 10:50-11:00 & Rest & \\
\hline 11:00-11:08 & Jump rope & Jumping rope with $45-50$ turns per min for $8 \mathrm{~min}$ \\
\hline $11: 10$ & End recording data & \\
\hline $12: 20$ & Leave the room & \\
\hline
\end{tabular}

Table 2 Sample Characteristics by Gender

\begin{tabular}{|c|c|c|c|c|c|c|c|c|c|c|c|c|}
\hline \multirow[b]{2}{*}{ Variable } & \multicolumn{4}{|c|}{ Male } & \multicolumn{4}{|c|}{ Female } & \multicolumn{4}{|c|}{ Total } \\
\hline & Mean & SD & Min & Max & Mean & SD & Min & Max & Mean & SD & Min & Max \\
\hline Age (yrs) & 12.41 & 0.32 & 12.03 & 12.99 & 12.51 & 0.35 & 12.08 & 13.18 & 12.45 & 0.33 & 12.03 & 13.18 \\
\hline Height $(\mathrm{cm})$ & $161.87 *$ & 8.20 & 144.70 & 171.90 & 155.58 & 3.79 & 148.90 & 162.50 & 159.35 & 7.40 & 144.70 & 171.90 \\
\hline Weight (kg) & $54.32 *$ & 11.42 & 34.20 & 77.10 & 45.63 & 6.37 & 39.00 & 54.50 & 50.84 & 10.52 & 34.20 & 77.10 \\
\hline BMI $\left(\mathrm{kg} / \mathrm{m}^{2}\right)$ & 20.62 & 3.64 & 15.85 & 30.31 & 18.81 & 2.22 & 16.05 & 23.01 & 19.90 & 3.23 & 15.85 & 30.31 \\
\hline Z-BMI & 0.22 & 1.13 & -1.25 & 3.22 & -0.34 & 0.69 & -1.19 & 0.96 & 0.00 & 1.00 & -1.25 & 3.22 \\
\hline $\begin{array}{l}\text { Resting HR (beats/ } \\
\text { min) }\end{array}$ & $80.41 * * *$ & 9.78 & 56.00 & 94.00 & 69.55 & 6.71 & 56.00 & 80.00 & 76.14 & 10.13 & 56.00 & 94.00 \\
\hline RMR (kcal/kg/h) & 1.42 & 0.26 & 0.99 & 1.83 & 1.34 & 0.25 & 1.04 & 1.92 & 1.39 & 0.25 & 0.99 & 1.92 \\
\hline BMR (kcal/kg/h) & 1.41 & 0.24 & 0.95 & 1.84 & 1.37 & 0.31 & 0.93 & 2.18 & 1.39 & 0.27 & 0.93 & 2.18 \\
\hline
\end{tabular}

$* P<.05$; *** $P<.005$; the significance markers refer to differences between gender.

Abbreviations: RMR, resting metabolic rate; BMR, basal metabolic rate. 
Table 3 Energy Expenditure and METs of Examined Activities

\begin{tabular}{|c|c|c|c|c|c|c|c|c|c|c|c|c|c|}
\hline \multirow[b]{2}{*}{ Variable } & \multicolumn{4}{|c|}{ Male } & \multicolumn{4}{|c|}{ Female } & \multicolumn{4}{|c|}{ Total } & \multirow{2}{*}{$\begin{array}{l}\text { METs from } \\
\text { Compendium }\end{array}$} \\
\hline & Mean & SD & Min & Max & Mean & SD & Min & Max & Mean & SD & Min & Max & \\
\hline \multicolumn{14}{|l|}{$\mathrm{EE}(\mathrm{kcal} / \mathrm{kg} / \mathrm{h})$} \\
\hline Sleep & 1.13 & 0.20 & 0.64 & 1.48 & 1.14 & 0.26 & 0.63 & 1.76 & 1.13 & 0.22 & 0.63 & 1.76 & \\
\hline Homework & 1.69 & 0.24 & 1.26 & 2.17 & 1.67 & 0.33 & 1.09 & 2.43 & 1.68 & 0.28 & 1.09 & 2.43 & \\
\hline Reading & 1.63 & 0.31 & 0.91 & 2.09 & 1.64 & 0.38 & 0.86 & 2.53 & 1.63 & 0.33 & 0.86 & 2.53 & \\
\hline TV & 1.59 & 0.26 & 1.18 & 2.13 & 1.57 & 0.33 & 0.91 & 2.30 & 1.58 & 0.29 & 0.91 & 2.30 & \\
\hline Computer Game & 1.55 & 0.24 & 1.06 & 1.93 & 1.55 & 0.32 & 0.98 & 2.31 & 1.55 & 0.27 & 0.98 & 2.31 & \\
\hline Radio Gymnastics & 4.21 & 0.67 & 2.89 & 5.27 & 3.70 & 1.19 & 1.75 & 6.79 & 4.00 & 0.93 & 1.75 & 6.79 & \\
\hline Jump Rope & 4.49 & 1.07 & 2.33 & 6.57 & 3.96 & 1.09 & 2.80 & 6.80 & 4.28 & 1.09 & 2.33 & 6.80 & \\
\hline \multicolumn{14}{|l|}{ MET } \\
\hline Sleep & 0.80 & 0.11 & 0.57 & 0.99 & 0.85 & 0.14 & 0.51 & 1.03 & $0.82 * * *$ & 0.12 & 0.51 & 1.03 & 0.9 \\
\hline Homework & 1.20 & 0.12 & 0.90 & 1.39 & 1.25 & 0.18 & 0.89 & 1.52 & $1.22 * * *$ & 0.15 & 0.89 & 1.52 & 1.4 \\
\hline Reading & 1.15 & 0.15 & 0.81 & 1.35 & 1.24 & 0.27 & 0.70 & 1.77 & $1.19 * *$ & 0.21 & 0.70 & 1.77 & 1.3 \\
\hline TV & 1.13 & 0.09 & 0.92 & 1.27 & 1.18 & 0.21 & 0.74 & 1.51 & 1.15 & 0.15 & 0.74 & 1.51 & 1.2 \\
\hline Computer Game & 1.10 & 0.10 & 0.95 & 1.26 & 1.16 & 0.19 & 0.80 & 1.56 & $1.13 * * *$ & 0.14 & 0.80 & 1.56 & 1.5 \\
\hline Radio Gymnastics & 3.01 & 0.45 & 2.36 & 3.81 & 2.75 & 0.62 & 1.43 & 3.53 & 2.91 & 0.53 & 1.43 & 3.81 & Not Available \\
\hline Jump Rope & 3.22 & 0.85 & 2.13 & 5.87 & 2.97 & 0.62 & 2.05 & 3.82 & $3.12 * * *$ & 0.77 & 2.05 & 5.87 & 6.2 \\
\hline
\end{tabular}

** $P<.01$; *** $P<.005$; the significance markers refer to differences between observed MET scores vs. Youth Compendium scores.

Abbreviations: EE, energy expenditure.

Interestingly, it is not clear how the standards were established. In our study, although we asked participants to perform 45 to 50 turns per minute for 8 minutes continuously, none of them could do this because they did not have skills to perform jump rope continuously. In fact, all the participants could only accumulate 45 to 60 turns per minute for 3 to 4 minutes-with many stops. However, even with stops and participants rating their effort as light, the METs of jump rope still reached moderate intensity level. Although this value is much lower than the Youth Compendium jump rope MET value (6.2), it should be noted that the MET value for jump rope with light effort was estimated and not measured in the Youth Compendium. ${ }^{9}$ How the effort was defined (ie, number of turns per minute) and/or race/ethnicity difference may account for the large difference between the MET value observed in this study and the value listed in the Youth Compendium. Given that jump rope is a school-based activity in which students engage on a regular basis, this finding has potential to increase the use of data to make future recommendations for promoting $\mathrm{PA}$ in China.

In conclusion, although the lack of diversity in terms of race/ ethnicity, age range and activities examined, along with the relatively small sample size may limit its generalizability, this study provides empirical evidence for more accurately assessing EE of activities typically performed by 12 -year-old Chinese youth. This study is also the first to determine MET values for radio gymnastics and jump rope, which provide not only a more solid foundation for developing future policies to promote school based physical activities, but also practical validation linking $\mathrm{EE}$ to jump rope national performance standards for sixth graders in China. ${ }^{8}$ Further research is needed to determine the METs of Chinese youth in different age ranges for a variety of activities using large samples.

\section{Acknowledgments}

This study was supported by the National Social Science Foundation of China (No.13BTY044) and Shanghai Key Laboratory of Human Sport Competence Development and Maintenances (Shanghai University of Sport: No.11DZ2261100).

\section{References}

1. Song Y, Ma J, Wang H, et al. Secular trends of obesity prevalence in Chinese children from 1985 to 2010: Urban-rural disparity. Obesity (Silver Spring). 2015;23(2):448-453. PubMed doi:10.1002/oby. 20938

2. World Health Organization. Global Recommendations on Physical Activity for Heath. 2010. http://www.who.int/dietphysicalactivity/ factsheet_young_people/en/. Accessed December 29, 2015.

3. General Administration of Sport of China. National Youth and Adult Physical Fitness Survey. 2015. http://www.sport.gov.cn/n16/n1077/ n297454/7299833.html. Accessed December 29, 2015.

4. Tudor-Locke C, Ainsworth B, Adair L, Du S, Popkin B. Physical activity and inactivity in Chinese school-aged youth: the China Health and Nutrition Survey. Int J Obes. 2003;27:1093-1099. PubMed doi:10.1038/sj.ijo.0802377

5. Chinese Department of Education. Notification of Implementing the Third Set of Nationwide Student Series of Radio Gymnastics. 2008. http://www.moe.gov.cn/srcsite/A17/moe_938/s3276/200807/ t20080707_80864.html. Accessed May 25, 2016.

6. Liu J, Xu Y, Ma R. 60 years of history: a review and prospects for the radio gymnastics in China. Sports Culture Guide. 2011;6:14-16. 
7. Li P, Xiao W. The feasibility of implementing a jump rope curriculum in Shanghai middle school students. Contemporary Sports Technology. 2013;3(20):104-105.

8. Yang G, Sheng Z. Chinese Student Fitness Healthy Zone National Standards: Interpretations. Beijing, China: People's Education Press; 2007.

9. Ridley K, Ainsworth B, Olds T. Development of a compendium of energy expenditures for youth. Int J Behav Nutr Phys Act. 2008;5(45):1-8. PubMed
10. Wouters-Adriaens M, Westerterp K. Low resting energy expenditure in Asians can be attributed to body composition. Obesity (Silver Spring). 2008;16(10):2212-2216. PubMed doi:10.1038/ oby. 2008.343

11. Weir JB. New methods for calculating metabolic rate with special reference to protein metabolism. J Physiol. 1949;109(12):1-9. PubMed doi:10.1113/jphysiol.1949.sp004363 